\title{
Synthesis of Chiral Heteroaryl-Substituted Dihydropyran Derivatives via Ring Closing Enyne Metathesis Reaction
}

\author{
Ayșegül Gümüş, Selçuk Gümüş, Nalan Nuriye Büyükadalı and Nezir Aslan \\ Van Yuzuncu Yil University, Faculty of Science, Department of Chemistry, Van, Turkey
}

\begin{abstract}
The ring closing enyne metathesis reactions have been applied to systems derived from homopropargylic alcohol backbones are described. The key intermediates 2benzofuranyl and 2-benzothiophenyl homopropargylic alcohols were synthesized from their corresponding carboxyaldehyde derivatives and resolved to give the corresponding enantiopure acetates and the alcohols with high ee values through enzymatic resolution. Then, enantiomerically enriched enyne skeletons derived from homopropargylic alcohols were subjected to the ring closing metathesis reaction via first generation Grubbs' catalysts and corresponding chiral heteroaryl-substituted dihydropyran derivatives were obtained with good yields.
\end{abstract}

\section{Introduction}

Dihydropyrans which are among the most investigated heterocyclic structural motifs in the past decade are widely occur in numerous natural and synthetic products. ${ }^{1}$ Many of these compounds show potential biological activities, such as cytotoxicity against some cancers, anti-infectivity and anti-HCV activities, and are widely used in pharmaceuticals. ${ }^{2}$ Some of the most widely used methods for the preparation of dihydropyrans are based on hetero-Diels-Alder cycloaddition, ${ }^{3}$ electrophile-initiated alkylation of glycals, ${ }^{4}$ olefin metathesis, ${ }^{5}$ dioxanone Claisen rearrangement ${ }^{6}$ and allylation-Prins cyclization. ${ }^{7}$

Ring closing metathesis (RCM) reaction is one of the most powerful tool for the synthesis of oxacyclic rings due to its mild experimental conditions and wide applicability in synthetic organic chemistry. ${ }^{8-9}$ Tremendous carbocyclic and heterocyclic rings have been elegantly constructed cycles in the presence of a metathesis catalyst, for example, first and second generation of Grubbs' ruthenium-based catalyst from relatively simple precursors by the RCM reaction. 
Benzothiophene and benzofuran molecules are found to be important scaffolds in synthetic medicinal chemistry. Their natural and synthetic derivatives display a wide spectrum of pharmacological activities like antioxidant, antibacterial, antimicrobial, antifungal, antiviral and anticancer activities etc [13-17]. Moreover substituted benzofurans find application such as of oxidant [18], antioxidants, fluorescent sensor [19], brightening agents, a variety of drugs and in other field of chemistry and agriculture [20].

Herein, we describe the chemoenzymatic synthesis of optically active heteroaryl substituted dihydropyrans starting from benzofuran and benzothiophene-substituted homopropargyl alcohols.

\section{Results and Discussion}

The parent benzofuranyl and benzothiophenyl homopropargylic alcohols 1a-b were synthesized by the addition of propargyl nucleophile to corresponding aldehydes and enzymatically resolved in our previous study [21]. Chiral heteroaryl-substituted homopropargylic alcohols are good candidates for the preparation of chiral enyne systems because of their $\mathrm{O}$-anchoring site. These enyne scaffolds are very valuable precursors for the construction of dihydropyran skeletons by ring closing metathesis reaction.

The enyne systems (-)-2a-b were first built on enantiomerically enriched homopropargylic alcohols (-)-1a-b (99\% ee for each) by O-allylation using allyl bromide in the presence of $\mathrm{NaH}$ and tetrabutylammonium iodide (TBAI) in THF (Scheme 1). Enyne scaffolds (-)-2a-b were subsequently subjected to ring closing enyne metathesis by employing Grubbs' first generation catalyst $(5 \mathrm{~mol} \%)$ at room temperature. Benzofuran and benzothiophene-substituted dihydropyran derivatives (-)-3a-b were isolated by $84 \%$ and $93 \%$ yields, respectively. 


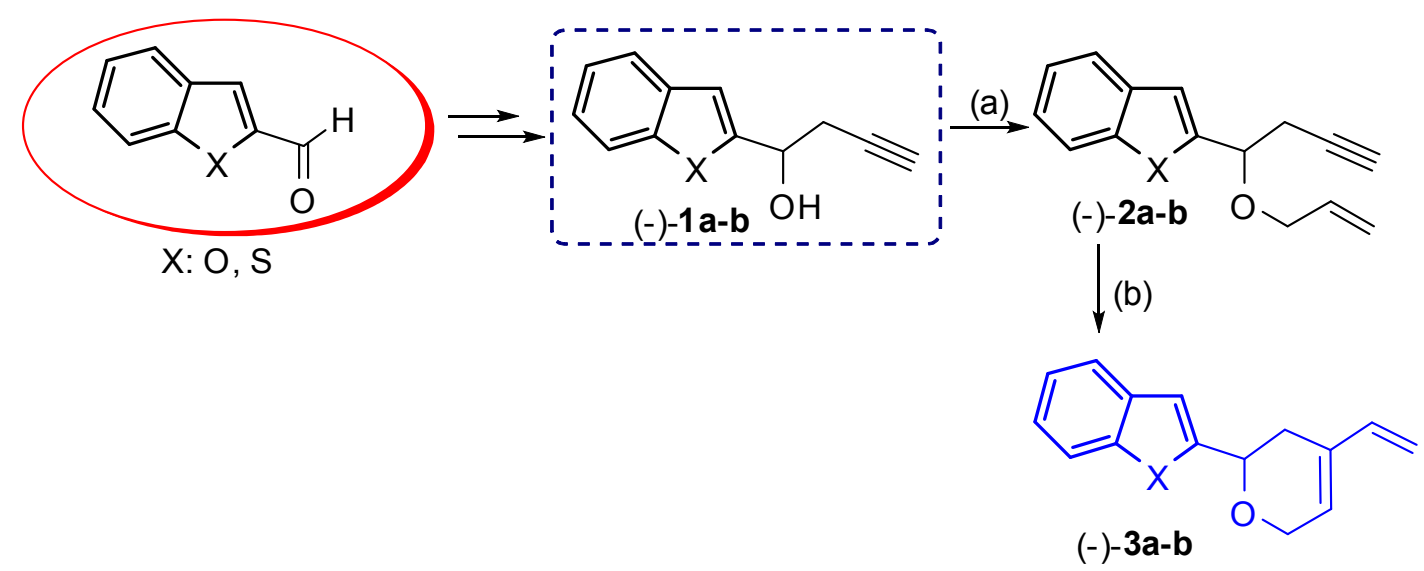

Scheme 1. Reagents and conditions: (a) allyl bromide, NaH, TBAI, THF; (b) Grubbs' 1st generation cat. $(5 \mathrm{~mol} \%)$.

\section{Conclusion}

In summary, we have described the design and synthesis of novel hybrid compounds between heteroaryl ring (benzofuran and benzothiophene) and dihydropyran scaffold. The enantiomerically enriched homopropargylic alcohols were converted to chiral enyne structures by $\mathrm{O}$-allylation method. Then, ring closing metathesis reaction was applied on these enynes. In conclusion, novel chiral heteroaryl-substituted dihydropyran derivatives were prepared in this study.

\section{Experimental}

\subsection{General}

All experiments were carried out in pre-dried glassware an inert atmosphere of argon. ${ }^{1} \mathrm{H}$ NMR and ${ }^{13} \mathrm{C}$ NMR spectra were recorded in $\mathrm{CDCl}_{3}$ on a Bruker Spectrospin Avance DPX-400 spectrometer. ${ }^{1} \mathrm{H}$ (400 MHz) and ${ }^{13} \mathrm{C} \mathrm{NMR}$ were recorded in $\mathrm{CDCl}_{3}$ and the chemical shifts are expressed in ppm relative to $\mathrm{CDCl}_{3}\left(\delta 7.26\right.$ and 77.0 for ${ }^{1} \mathrm{H}$ and ${ }^{13} \mathrm{C} \mathrm{NMR}$, respectively) as the internal standard. Optical rotations were measured in a $10 \mathrm{~cm}$ cell using a Rudolph Research, Autopol III polarimeter. HRMS spectra were recorded on an Waters SYNAPT G1 MS (ESI-TOF-MS) at METU Central Laboratory R\&D Training and Measurement Center. 
Flash column chromatography was performed bu using thick-walled glass columns and silica gel (60-mesh; Merck). The reactions were monitored by thin-layer chromatography (TLC) using Merck 0.2-mm silica gel 60 F254 analytical aluminum plates, visualized by UV light. All extracts were dried over anhydrous magnesium sulfate and solutions were concentrated under reduced pressure by using a rotary evaporator.

\subsection{General procedure for the synthesis of enyne derivatives (-)-2a-b.}

To a solution of (-)-1a-b in dry THF (10 mL) was added NaH (96 mg, 60\% dispersion in mineral oil, $2.4 \mathrm{mmol}$ ) under argon. The solution was stirred until $\mathrm{H}_{2}$ gas removal was complete (approximately $30 \mathrm{~min}$ ). Next, allyl bromide added dropwise followed by tetrabutylammonium iodide $(2 \mathrm{mmol})$. The mixture was stirred for an additional $2 \mathrm{~h}$ and hydrolyzed by cautious addition of water $(20 \mathrm{~mL})$. The aqueous layer was extracted with ether $(3 \times 20 \mathrm{~mL})$. The combined organic phase was dried over $\mathrm{MgSO}_{4}$ and evaporated in vacuo. The crude product mixtures were purified by flash column chromatography using ethyl acetate/ hexane (1:7) as the eluent.

\subsection{1. (-)-2-(1-(allyloxy)but-3-ynyl)benzofuran, (-)-2a}

Yellow oil. (0.44 g, 98\% yield); $[\alpha]_{\mathrm{D}}{ }^{16}=-54.8\left(\mathrm{c} 0.5, \mathrm{CH}_{2} \mathrm{Cl}_{2}\right) ;{ }^{1} \mathrm{H} \mathrm{NMR}\left(\mathrm{CDCl}_{3}, 400 \mathrm{MHz}\right): \delta$ 7.58-7.56 (m, 1H), 7.51-7.48 (m, 1H), 7.32-7.21 (m, 2H), $6.74(\mathrm{~s}, 1 \mathrm{H}), 5.97-5.87(\mathrm{~m}, 1 \mathrm{H})$, 5.34-5.28 (m, 1H), 5.23-5.20 (m, 1H), $4.68(\mathrm{t}, J=6.8 \mathrm{~Hz}, 1 \mathrm{H}), 4.14-4.09(\mathrm{~m}, 1 \mathrm{H}), 4.02-3.97$ $(\mathrm{m}, 1 \mathrm{H}), 2.88(\mathrm{dd}, J=2.6$ and $6.8 \mathrm{~Hz}, 2 \mathrm{H}), 1.99(\mathrm{t}, J=2.6 \mathrm{~Hz}, 1 \mathrm{H}) ;{ }^{13} \mathrm{C} \mathrm{NMR}\left(\mathrm{CDCl}_{3}, 400\right.$ $\mathrm{MHz}): \delta 155.3,155.0,134.1,127.8,124.4,122.8,121.1,117.8,111.5,105.4,79.9,72.9,70.3$, 70.2, 24.6. HRMS (ESI-TOF). Anal. Calcd for $\mathrm{C}_{15} \mathrm{H}_{14} \mathrm{O}_{2}[\mathrm{M}+\mathrm{Na}]^{+}: \mathrm{m} / z$ 249.0891. Found: $\mathrm{m} / \mathrm{z}$ 249.0879 .

\subsection{2. (-)-2-(1-(allyloxy)but-3-ynyl)benzo[b]thiophene, (-)-2b}

Yellow oil. (0.46 g, 95\% yield); $[\alpha]_{\mathrm{D}}{ }^{16}=-36.0\left(\mathrm{c} 0.5, \mathrm{CH}_{2} \mathrm{Cl}_{2}\right) ;{ }^{1} \mathrm{H} \mathrm{NMR}\left(\mathrm{CDCl}_{3}, 400 \mathrm{MHz}\right): \delta$ 7.85-7.83 (m, 1H), 7.77-7.75 (m, 1H), 7.39-7.31 (m, 2H), $7.29(\mathrm{~s}, 1 \mathrm{H})$, 5.99-5.89 (m, 1H), 5.35-5.29 (m, 1H), 5.25-5.21 (m, 1H), $4.85(\mathrm{td}, J=1.6$ and $6.7 \mathrm{~Hz}, 1 \mathrm{H}), 4.19-4.10(\mathrm{~m}, 1 \mathrm{H})$, 4.00-3.94 (m, 1H), 2.92-2.85 (m, 1H), 2.79-2.72 (m, 1H), 2.04 (q, J=2.7 Hz, 1H); ${ }^{13} \mathrm{C} \mathrm{NMR}$ 
$\left(\mathrm{CDCl}_{3}, 400 \mathrm{MHz}\right): \delta 145.3,139.7,139.2,134.1,124.4,124.3,123.6,122.6,122.5,117.7$, 80.1, 75.5, 70.7, 70.0, 28.2. HRMS (ESI-TOF). Anal. Calcd for $\mathrm{C}_{15} \mathrm{H}_{14} \mathrm{OS}[\mathrm{M}+\mathrm{H}]^{+}: \mathrm{m} / \mathrm{z}$ 243.0843. Found: $m / z 243.0851$.

\subsection{Ring closing metathesis reactions}

O-Allyl anchored substrates (-)-2a-b $(0.2 \mathrm{mmol})$ were dissolved in DCM (10 mL) and Grubbs' first generation catalyst $(5 \mathrm{~mol} \%)$ was added to the solution. The reaction was monitored by TLC. The crude product was concentrated and purified by short column chromatography.

\subsection{1. (-)-2-(4-vinyl-3,6-dihydro-2H-pyran-2-yl)benzofuran, (-)-3a}

Yellow oil. (0.42 g, 93\% yield); $[\alpha]_{\mathrm{D}}{ }^{20}=-47.6\left(\mathrm{c} 0.5, \mathrm{CH}_{2} \mathrm{Cl}_{2}\right) ;{ }^{1} \mathrm{H} \mathrm{NMR}\left(\mathrm{CDCl}_{3}, 400 \mathrm{MHz}\right): \delta$ 7.58-7.56 (m, 1H), 7.52-7.50 (m, 1H), 7.31-7.21 (m, 2H), 6.73-6.72 (m, 1H), 6.48-6.41 (dd, $\mathrm{J}=10.7$ and $17.5 \mathrm{~Hz}, 1 \mathrm{H}), 5.82-5.81(\mathrm{~m}, 1 \mathrm{H}), 5.22(\mathrm{~d}, J=7.5 \mathrm{~Hz}, 1 \mathrm{H}), 5.08(\mathrm{~d}, J=9.7 \mathrm{~Hz}, 1 \mathrm{H})$, $4.84(\mathrm{dd}, J=4.0$ and $9.6 \mathrm{~Hz}, 1 \mathrm{H}), 4.47-4.44(\mathrm{~m}, 2 \mathrm{H}), 2.76-2.67(\mathrm{~m}, 1 \mathrm{H}), 2.63-2.57(\mathrm{~m}, 1 \mathrm{H})$;

${ }^{13} \mathrm{C} \mathrm{NMR}\left(\mathrm{CDCl}_{3}, 400 \mathrm{MHz}\right): \delta 156.7,154.9,137.8,132.9,127.9,126.2,124.3,122.8,121.1$, 111.9, 111.4, 103.6, 69.3, 65.8, 27.7. HRMS (ESI-TOF). Anal. Calcd for $\mathrm{C}_{15} \mathrm{H}_{14} \mathrm{O}_{2}[\mathrm{M}+\mathrm{H}]^{+}$: $m / z$ 227.1072. Found: $m / z$ 227.1079.

\subsection{2. (-)-2-(benzo[b]thiophen-2-yl)-4-vinyl-3,6-dihydro-2H-pyran, (-)-3b}

Yellow solid. (0.44 g, 90\% yield); mp 49-51 ${ }^{\circ} \mathrm{C} .[\alpha]_{\mathrm{D}}{ }^{20}=-51.7\left(\mathrm{c} 0.5, \mathrm{CH}_{2} \mathrm{Cl}_{2}\right) ;{ }^{1} \mathrm{H}$ NMR $\left(\mathrm{CDCl}_{3}, 400 \mathrm{MHz}\right): \delta 7.84(\mathrm{~d}, J=7.5 \mathrm{~Hz}, 1 \mathrm{H}), 7.74$ (d, $\left.J=7.2 \mathrm{~Hz}, 1 \mathrm{H}\right), 7.36-7.29(\mathrm{~m}, 2 \mathrm{H}), 6.44$ (dd, $J=10.7$ and $17.4 \mathrm{~Hz}, 1 \mathrm{H}), 5.81(\mathrm{~s}, 1 \mathrm{H}), 5.21(\mathrm{~d}, J=17.5 \mathrm{~Hz}, 1 \mathrm{H}), 5.07$ (d, $J=10.7 \mathrm{~Hz}, 1 \mathrm{H})$,

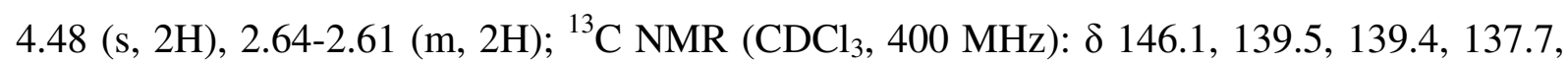
133.1, 126.3, 124.2, 124.1, 123.5, 122.4, 120.2, 111.8, 71.9, 66.1, 31.3. HRMS (ESI-TOF). Anal. Calcd for $\mathrm{C}_{15} \mathrm{H}_{15} \mathrm{OS}[\mathrm{M}+\mathrm{H}]^{+}: m / z$ 243.0844. Found: $m / z$ 243.0850.

\section{Acknowledgements}

We are grateful to Presidency of Scientific Research Projects of Van Yuzuncu Yil University for financial support (FYL-2017-5946). 


\section{References}

1. (a) Gupta, K.C.; Gupta, P.; Singh, P.; Singh, S.V.; Agarwal, S. Chemistry of natural dyes; Daniel, M., Ed. Natural Dyes: Scope and Challenges. Scientific: Jodhpur, India, 2006; pp. 734; (b) Marco, J.A.; Carda, M.; Murga, J.; Falomir, E. Tetrahedron 2007, 63, 2929-2958; (c) Rodriguez, R.; Moses, J.E.; Adlington, R.M.; Baldwin, J.E. Org. Biomol. Chem. 2005, 3, 3488-3495; (d) Cheung, A.K.; Murelli, R.; Snapper, M.L. J. Org. Chem. 2004, 69, 57125719.

2. (a) Apparao, S.; Maier, M.E.; Schmidt, R.R. Synthesis 1987, 900-904; (b) Marmsaeter, F. P.; West, F.J. J. Am. Chem. Soc. 2001, 123, 5144-5145; (c) Xu, Y.C. Recent Res. Dev. Org. Chem. 2000, 4, 423-441; (d) Cheung, A.K.; Snapper, M.L. J. Am. Chem. Soc. 2002, 124, 11584-11585; (e) Hatakeyama, S.; Ochi, N.; Numata, H.; Takano, S. Chem. Commun. 1988, 1202-1204; (f) Hojo, M.; Masuda, R.; Sakaguchi, S.; Takagawa, M. Synthesis 1986, 10161017.

3. (a) Danishefsky, S.J.; DeNinno, M.P. Angew. Chem., Int. Ed. 1987, 26, 15-23; (b) Danishefsky, S.J.; Selnick, H.G.; Zelle, R.E.; DeNinno, M.P. J. Am. Chem. Soc. 1988, 110, 4368-4378; (c) Lubineau, A.; Auge, J.; Lubin, N. Tetrahedron 1993, 49, 4639-4650; (d) Huang, H.; Panek, J.S. J. Am. Chem. Soc. 2000, 122, 9836-9837; (e) Evans, D.A.; Johnson, J. S.; Othava, E.J. J. Am. Chem. Soc. 2000, 122, 1635-1649; (f) Deligny, M.; Carreaux, F.; Toupet, L.; Carboni, B. Adv. Synth. Catal. 2003, 345, 1215-1219; (g) Deligny, M.; Carreaux, F.; Carboni, B.; Toupet, L.; Gilles Dujardin, G. Chem. Commun. 2003, 276-277.

4. Steinhuebel, D.P.; Fleming, J. J.; Du Bois, J. Org. Lett. 2002, 4, 293-295.

5. (a) Takai, K.; Okazoe, T.; Oshima, K.; Utimoto, K. J. Org. Chem. 1987, 52, 4412-4414; (b) Grubbs, R.H.; Fujimura, O.; Fu, G.C. J. Org. Chem. 1994, 59, 4029-4031; (c) Clark, J.S.; Kettle, J.G. Tetrahedron Lett. 1997, 38, 123-126; (d) Clark, J. S.; Kettle, J. G. Tetrahedron 1999, 55, 8231-8248.

6. Sherry, B.D.; Maus, L.; Laforteza, B.N.; Toste, F.D. J. Am. Chem. Soc. 2006, 128, 81328133.

7. (a) Viswanathan, G.S.; Yang, J.; Li, C.-J. Org. Lett. 1999, 1, 993-995; (b) Dobbs, A.P.; Martinovic, S. Tetrahedron Lett. 2002, 43, 7055-7057.

8. For the reviews on RCM reactions, see: (a) Furstner, A. Angew. Chem., Int. Ed. 2000, 39, 3013-3043; (b) Trnka, T.M.; Grubbs, R.H. Acc. Chem. Res. 2001, 34, 18-29; (c) Fürstner, A., Ed. Alkene Metathesis in Organic Synthesis. Springer: Berlin, 1998. 
9. For the synthesis of heterocyclic compounds by RCM reaction, (a) Deiters, A.; Martin, S. F. Chem. Rev. 2004, 104, 2199-2238; (b) Kim, J.M.; Lee, K.Y.; Lee, S.; Kim, J.N. Tetrahedron Lett. 2004, 45, 2805-2808.

10. Ho, Y.S.; Duh, J.S.; Jeng, J.H.; Wang, Y.J.; Liang, Y.; Lin, C.H.; Tseng, C.J.; Yu, C.F.; Chen, R.J.; Lin, J.K. Int. J. Cancer 2001, 91, 393.

11. Marquès, S.; Buchet, R.; Popowycz, F.; Lemaire, M.; Mebarek, Saïda Bioorg. Med. Chem. Lett. 2016, 26, 1457.

12. Hoang, D.M.; Ngoc, T.M.; Dat, N.T.; Ha, D.T.; Kim, Y.H.; Luong, H.V.; Ahn, J.S.; Bae, K. Bioorg. Med. Chem. Lett. 2009, 19, 6759.

13. Isloor, A.M.; Kalluraya, B.; Pai, K.S. Eur. J. Med. Chem. 2010, 45, 825-830.

14. Pinto, E.; Queiroz, M.J.; Vale-Silva, L.A.; Oliveira, J.F.; Begouin, A.; Begouin, J.M.; Kirsch, G. Bioorg Med Chem 2008, 16, 8172-8177.

15. Androsov, D.A.; Solovyev, A.Y.; Petrov, M.L.; Butcher, R.J.; Jasinski, J.P. Tetrahedron 2010, 66, 2474-2485.

16. Dit Chabert, J.F.; Marquez, B.; Neville, L.; Joucla, L.; Broussous, S.; Bouhours, P. Bioorg. Med. Chem. 2007, 15, 4482-4497.

17. Boateng, C.A.; Eyunni, S.V.K.; Zhu, X.U.; Etukala, J.R.; Bricker, B.A.; Ashfaq, M.K. Bioorg. Med. Chem. 2011, 19, 458-470.

18. Karatas, F.; Koca, M.; Kara, H.; Servi, S. Synthesis and oxidant properties of novel (5bromobenzofuran-2-yl)(3-methyl-3-mesitylcyclobutyl) ketonethiosemicarbazone, Eur. J. Med. Chem. 2006, 41, 664-669.

19. Oter, O.; Ertekin, K.; Kirilmis, C.; Koca, M.; Ahmedzade, M. Characterization of a newly synthesized fluorescent benzofuran derivative and usage as a selective fiber optic sensor for Fe(III), Sens. Actuators B: Chem. 2007, 122, 450-456.

20. Habermann, J.; Ley, S.V.; Smits, R. Three-step synthesis of an array of substituted benzofurans using polymer-supported reagents, J. Chem. Soc. Perkin Trans. 1999, 1, 24212423.

21. Büyükadal1, N. N.; Seven, S.; Aslan, N.; Yenidede, D.; Gümüş, A. Tetrahedron-Asymm. 2015, 26, 1285. 